\title{
Sparse Preserving Feature Weights Learning
}

\author{
Guangsheng Xia, Hui Yan*, Jian Yang \\ School of Computer Science and Engineering, \\ Nanjing University of Science and Technology, \\ 210094, China
}

\begin{abstract}
In this paper, we propose a novel unsupervised feature selection algorithm, named sparse preserving feature weights learning (SPFW), which is based on the recent local data representation theory, sparse representation. SPFW differs from traditional feature selection algorithms in two aspects: (1) SPFW is designed on the locality measurement criterion with sparse reconstruction residual minimization. It adaptively determines the locality based on sparse representation, instead of fixing the $k$-nearest neighbors in the original feature space. (2) SPFW selects the most discriminative feature subset from the whole feature set in batch mode, instead of selecting features individually. To optimize the proposed formulation, we propose an efficient iterative algorithm, where each iteration reduces to a subproblem which can be solved with some off-the-shelf toolboxes. We conduct experiments on two face datasets to evaluate the performance of feature selection in terms of classification and clustering, which demonstrate the effectiveness of the proposed algorithm.
\end{abstract}

Keywords: Joint feature selection, Sparse representation, Feature weights learning

\section{Introduction}

Data are often represented as high-dimensional feature vectors in many applications, such as text processing [1], gene expression analysis [2], and face recognition [3]. However, features are often correlated or redundant to each other, and they may

${ }^{*}$ Corresponding author:

Tel.: +86 025 84317297-3038.

Email address: yanhui@njust.edu.cn

Preprint submitted to Neurocomputing

September 14, 2015

(C) 2016. This manuscript version is made available under the Elsevier user license http://www.elsevier.com/open-access/userlicense/1.0/ 
even be corrupted by noises. Usually only a small set of features are really important. Consequently, it is necessary to reduce dimensionality, which can be achieved by feature selection or feature extraction. Feature selection seeks the most informative subset of features from original feature set, while feature extraction transforms an original high-dimensional feature space to a low-dimensional space by combining original features. Compared with feature extraction, feature selection improves result comprehensibility.

During recent years, feature selection has drawn increasing attention, and many feature selection algorithms have been proposed. According to the availability of label information, feature selection can be classified into two main families: unsupervised [4], [5], [6] and supervised feature selection algorithms [7], [8], [9], [10]. Supervised feature selection algorithms can mine the correlation between features and data labels that will guide the search for discriminative features. However, in many data mining applications, data labels are absent. Therefore, unsupervised feature selection is indispensable and it is considered to be much harder than supervised scenario. In this paper we focus on unsupervised feature selection.

Unsupervised feature selection algorithms frequently use data similarity preserving as measurement criterion [11] to assess the importance of features. For instance, Laplacian Score [12] first constructs a $k$-nearest neighbor graph. Then it computes scores for each feature according to their abilities of preserving nearest neighbor relationship in the original feature space. Similarly, [13] computes sparse score for each feature on the basis of sparse representation graph. Then they [12], [13] select the features one by one with the lowest feature scores.

Traditional locality preserving based feature selection algorithms have two widely acknowledged limitations.

1. Similarity is characterized by $k$-nearest neighbor relationship defined in original feature space, which may be inconsistent with the one in the space of selected features [14]. Therefore, the output of feature subset is suboptimal. Moreover, the optimal number of nearest neighbors, i.e., $k$, depends on datasets and so far how to determine the value of $k$ is still not solved. 
2. Greedy feature selection algorithms compute importance for each feature independently. The limitation is that the correlation and redundancy among features are neglected.

The sparse regularization model based algorithms have been proposed to address the second problem. Multi-cluster feature selection (MCFS) [15] proposes a two-stage approach, i.e., spectral regression and $l_{1}$-norm minimization, for feature selection. Joint embedding learning and sparse regression (JELSR) [16] performs the embedding learning and sparse regression jointly. [17] proposes a robust feature selection method, which jointly improves the robustness of graph embedding and sparse spectral regression. More recently, Nie [10] and Yang [6] apply $l_{2,1}$-norm regularization model to evaluate the importance of the selected features jointly. The $l_{2,1}$-norm minimization term makes feature selection matrix sparse in rows for the sake of feature selection.

These efforts confirm that it is more effective to select features jointly. Note that these feature selection matrices are not constrained to be nonnegative. Strictly speaking these algorithms belong to sparse feature extraction, not feature selection.

\subsection{Our contribution}

In this paper, we introduce sparse representation [18], [19] based measurement criterion and joint feature weights learning into a novel unsupervised feature selection method, which is named sparse preserving feature weights learning. Our objective minimizes sparse representation reconstruction residuals in the space of weighted features. Its appealing characteristics are summarized as follows:

- The proposed algorithm selects features with the abilities of preserving local geometric structure. Compared with traditional locality preserving feature selection methods, our proposed algorithm adaptively determines the locality based on sparse representation in the weighted space, instead of fixing the neighboring samples based on pairwise Euclidean distance, which is very sensitive to data noises, in the original space.

- The proposed algorithm selects the optimal feature subset in batch mode by learning a nonnegative constrained feature weight vector, instead of simply adding 
each feature into the feature pool separately. An effective and efficient iterative algorithm is developed, where each iteration reduces to a subproblem which can be solved with some off-the-shelf toolboxes.

The remainder of this paper is organized as follows. In Section 2, we briefly review sparse representation. We present our sparse preserving feature weights learning in Section 3. The experiments on two face datasets are completed in Section 4. Finally, we draw a conclusion in Section 5.

\section{Sparse representation}

Let $A=\left[a_{1}, \ldots, a_{n}\right] \in R^{m \times n}$ be the matrix composed of $n$ training samples with $C$ classes, where $m$ is the number of features. Given a test sample $y \in R^{m}$ from the $c$-th class, intuitively $y$ could be well approximated by the linear combination of the training samples from the $c$-th class. Based on this assumption, sparse representation aims to represent a given test sample as a sparse linear combination of all training samples, and the nonzero representation coefficients should concentrate on the training samples with the same class as the test sample.

Mathematically we represent $y$ in an overcomplete dictionary whose basis vectors are training sample themselves, i.e.,

$$
y=A \alpha
$$

where $\alpha \in R^{n}$ is the representation coefficient vector.

Under the assumption, $m<n$, the linear equation (1) is underdetermined, and this representation is naturally sparse. The sparsest solution of $\alpha$ can be sought by the following $l_{1}$-norm minimization problem [20], [21] :

$$
\hat{\alpha}=\arg \min \|\alpha\|_{1}, \text { s.t., } y=A \alpha
$$

where $\|\cdot\|_{1}$ denotes the $l_{1}$-norm. This problem (2) can be solved in polynomial time by standard linear programming algorithms [22] or recently proposed homotopy algorithm [23] . 


\section{Sparse preserving feature weights learning}

In this section we first present sparse preserving feature weights learning (SPFW) algorithm for unsupervised feature selection, and then put forward an iterative algorithm to solve the proposed objective function.

\subsection{Basic idea and formulation}

Feature subset selection can be viewed as a binary optimization problem, and this combinatorial search is often non-deterministic polynomial-time (NP) hard [24]. To some extent we alleviate this computational issue by using a joint feature weights learning strategy [25], [26]. Let $X=\left[x_{1}, \ldots, x_{n}\right] \in R^{d \times n}$ be the training data matrix in the original feature space. In the weighted feature space, parameterized by a nonnegative vector $w=\left[w_{1}, \ldots, w_{d}\right]^{T} \in R_{+}^{d}$, the feature vector of the $i$-th sample weighted by $w$ can be denoted as $\operatorname{diag}(w) x_{i}$, where $\operatorname{diag}(w) \in R^{d \times d}$ is a diagonal matrix with $w$ on the diagonal.

According to the sparse preserving decision rule [27], smaller sparse reconstruction residuals will lead to better classification results in general. Therefore, we choose to minimize the sparse reconstruction residuals in the weighted feature space as follows:

$$
\begin{aligned}
\min _{w,\left\{\alpha_{i}, i=1, \ldots, n\right\}} & \sum_{i=1}^{n}\left\|\operatorname{diag}(w)\left(x_{i}-X_{i} \alpha_{i}\right)\right\|_{F}^{2}+\beta\left\|\alpha_{i}\right\|_{1} \\
\text { s.t., } & \sum_{j=1}^{d} w_{j}=1, w_{j} \geq 0
\end{aligned}
$$

where $X_{i}=\left[x_{1}, \ldots, x_{i-1}, 0, x_{i+1}, \ldots, x_{n}\right] \in R^{d \times n}$ whose $i$-th column is all-zero vector, $\alpha_{i}$ is the sparse representation coefficient vector for the $i$-th training sample. $X_{i} \alpha_{i}$ is the sparse reconstruction of $x_{i}$ with the rest training samples. SPFW is similar as our latest work [28] because both have the same feature search strategy, which learns feature weights jointly in an unsupervised way. However, their feature measurements are different. SPFW introduces sparse representation into feature selection; [28] combines feature selection and adaptive neighbors preservation into a single framework. 


\subsection{Optimization}

Since in the objective function (3), $\left\{\alpha_{i}\right\}$ and $w$ are dependent on each other, it can not have a closed-form solution. Consequently, we update the variables alternately with others fixed by iteratively solving Eq.(3).

\subsubsection{Initialize $\left\{\alpha_{i}\right\}$}

A good initialization of $\left\{\alpha_{i}\right\}$ can speed up the convergence of the optimization and lead to a robust solution. For each training sample $x_{i}(i=1, \ldots, n)$ in the original feature space, we represent it using the remaining training samples. Its corresponding sparse representation coefficient vector $\alpha_{i}$ is calculated by solving the $l_{1}$-norm optimization problem.

$$
\alpha_{i}=\arg \min \left\|\alpha_{i}\right\|_{1} \text {, s.t., } x_{i}=X_{i} \alpha_{i}
$$

\subsubsection{Update $w$ as given $\left\{\alpha_{i}\right\}$}

By fixing $\left\{\alpha_{i}\right\}$ and removing the irrelevant terms in Eq.(3), we get the following optimization problem with respect to $w$ :

$$
\begin{array}{ll}
\min _{w} & \sum_{i=1}^{n}\left\|\operatorname{diag}(w)\left(x_{i}-X_{i} \alpha_{i}\right)\right\|_{F}^{2} \\
\text { s.t., } & \sum_{j=1}^{d} w_{j}=1, w_{j} \geq 0
\end{array}
$$

Let $\alpha^{w}=\left[\alpha_{1}, \ldots, \alpha_{n}\right]$ and $Y^{w}=X-X \alpha^{w}$, the objective function in the above optimization problem can be represented in the following matrix form:

$$
\begin{aligned}
& \sum_{i=1}^{n}\left\|\operatorname{diag}(w)\left(x_{i}-X_{i} \alpha_{i}\right)\right\|_{F}^{2} \\
= & \left\|\operatorname{diag}(w)\left(X-X \alpha^{w}\right)\right\|_{F}^{2} \\
= & \operatorname{Tr}\left(\operatorname{diag}^{2}(w) Y^{w} Y^{w T}\right)
\end{aligned}
$$


Finally, Eq.(5) is convert to the following problem:

$$
\begin{array}{ll}
\min & \sum_{j=1}^{d} w_{j}^{2}\left(\sum_{i=1}^{n} Y_{j i}^{w^{2}}\right) \\
\text { s.t., } & \sum_{j=1}^{d} w_{j}=1, w_{j} \geq 0
\end{array}
$$

This is a typical quadratic programming [29], which can be solved by the active set algorithm [30].

\subsubsection{Update $\left\{\alpha_{i}\right\}$ as given $w$}

By fixing $w$ and removing the irrelevant terms in Eq.(3), we get the following optimization problem with respect to all $\alpha_{i} \mathrm{~s}$ :

$$
\min _{\left\{\alpha_{i}\right\}} \sum_{i=1}^{n}\left\|\operatorname{diag}(w)\left(x_{i}-X_{i} \alpha_{i}\right)\right\|_{F}^{2}+\beta\left\|\alpha_{i}\right\|_{1}
$$

Since Eq.(8) is independent among different $i$, we can solve the following problem alternately for each $\alpha_{i}$ :

$$
\min _{\alpha_{i}}\left\|\operatorname{diag}(w)\left(x_{i}-X_{i} \alpha_{i}\right)\right\|_{F}^{2}+\beta\left\|\alpha_{i}\right\|_{1}
$$

Denote $L\left(\alpha_{i}\right)=\left\|\operatorname{diag}(w)\left(x_{i}-X_{i} \alpha_{i}\right)\right\|_{F}^{2}$. In Eq.(9), the first term $L\left(\alpha_{i}\right)$ is smooth, while in the second term $\left\|\alpha_{i}\right\|_{1}$ is non-smooth. $\beta$ is the regularization parameter. Then, we aim to minimize Eq.(9) with a two-step strategy [26] in each iteration:

- Step 1: Update $\hat{\alpha}_{i}$ via the following formulation:

$$
\hat{\alpha}_{i}=\arg \min _{\alpha_{i}}\left\|\operatorname{diag}(w)\left(x_{i}-X_{i} \alpha_{i}\right)\right\|_{F}^{2}
$$

The solution of (10) is given via subgradient descent as:

$$
\hat{\alpha}_{i}=\alpha_{i}-\lambda \frac{d\left(L\left(\alpha_{i}\right)\right)}{d\left(\alpha_{i}\right)}
$$

where $\lambda$ is the step size and set to be $\frac{1}{t} \cdot \frac{d\left(L\left(\alpha_{i}\right)\right)}{d\left(\alpha_{i}\right)}=2\left(X_{i}^{w}\right)^{T}\left(-x_{i}^{w}+X_{i}^{w} \alpha_{i}\right)$, where $X_{i}^{w}=\operatorname{diag}(w) X_{i}$, and $x_{i}^{w}=\operatorname{diag}(w) x_{i}$. 
- Step 2: Update $\alpha_{i}$, which is the approximation of $\hat{\alpha_{i}}$ under the $l_{1}$-norm minimization constraint.

$$
\alpha_{i}=\arg \min _{\alpha_{i}} \frac{1}{2}\left\|\alpha_{i}-\hat{\alpha_{i}}\right\|_{F}^{2}+\beta \lambda\left\|\alpha_{i}\right\|_{1}
$$

The solution of Eq.(12) is given by [31]

$$
\alpha_{i}=\left(\left|\hat{\alpha}_{i}\right|-\beta \lambda\right)_{+} \bigotimes \operatorname{sgn}\left(\hat{\alpha}_{i}\right)
$$

where $(a)_{+}=\max (0, a), \operatorname{sgn}(a)$ returns the sign of $a$, and $\bigotimes$ is the Hadamard operator. Note that any element in $\alpha_{i}$ less than $\beta \lambda$ will be set as zero, and this leads to a sparse solution for $\alpha_{i}$. The complete proposed algorithm is described in Algorithm 1.

Input: Data $X=\left[x_{1}, . ., x_{n}\right] \in R^{d \times n}$, the maximum iteration number $T$, and the regularization parameter $\beta$

Initial: Compute $\alpha_{i}^{0}$ via Eq.(4)

1. for $t=1, \ldots, T$ do

2. Update the feature weights vector $w^{t}$ by fixing $\alpha_{i}^{t-1}$ via Eq.(7)

3. for $i=1, \ldots, n$ do

4. $\quad$ Update the $i$-th sparse representation $\alpha_{i}^{t}$ via Eq.(11) and Eq.(13)

by fixing $w^{t}$ and other $\alpha_{j}^{t}(j \neq i)$

5. end for

6. end for

Output: $w^{t}$ and $\left\{\alpha_{i}^{t}\right\}$

Algorithm 1: Sparse preserving feature weights learning (SPFW)

\section{Experiments and analysis}

In this section, we first test the performance of the proposed algorithm with some state-of-the-art methods for unsupervised feature selection in terms of classification and clustering. And then we study the convergence performance of SPFW.

\subsection{Experimental data and setting}

140

We compare the proposed algorithm with some state-of-the-art unsupervised feature selection algorithms, including Max Variance, Laplacian Score, MCFS, unsu- 
pervised discriminative feature selection (UDFS) [6], and All Features as a baseline method:

All Features: Feature selection using all features.

Max Variance: Select features corresponding to the maximum variance.

Laplacian Score: Select features with the power of locality preserving.

MCFS: Select features using spectral embedding, followed by sparse coefficient vectors learning.

UDFS: Discriminative feature selection with $l_{2,1}$-norm regularization.

The experiments are conducted on two publicly available face image datasets, i.e., Extended YaleB dataset [32] and PIE dataset [33]. Extended YaleB dataset contains 38 subjects under 9 poses and 64 illumination conditions. We use a subset of Extended YaleB dataset in our experiments ${ }^{1}$, which has around 64 near frontal images under different illuminations per subject. PIE dataset contains 68 subjects with over 40000 face images under varying poses, illuminations, and expressions. We use one near frontal pose, i.e., PIE Pose C27 [34], with 2856 images. All images are manually aligned, cropped, and resized to be $32 \times 32$ pixels in our experiments. Fig.1 (a), (b) show the samples of cropped images on Extended YaleB and PIE respectively.

There are some parameters to be set in advance. Following Ref.[6], we set the number of nearest neighbors as 5 for Laplacian Score, MCFS and UDFS. For SPFW and UDFS we need to tune their regularization parameters. For a fair comparison, we tune these parameters from $\left\{10^{-5}, 10^{-4}, 10^{-3}, 10^{-2}, 10^{-1}, 1\right\}$. The numbers of selected features are set as $\{10,20, \ldots, 490,500\}$ for all datasets. And we pre-process the face images by scaling features (pixel values) to $[0,1]$.

\footnotetext{
${ }^{1}$ http://www.cad.zju.edu.cn/home/dengcai/Data/FaceData.html
} 


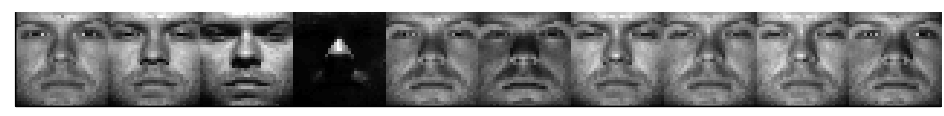

(a) Extended YaleB

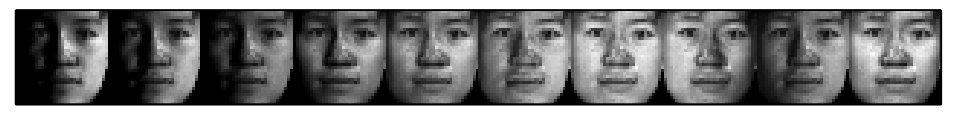

(b) PIE

Figure 1: Samples of cropped images of one person: (a) Extended YaleB (b) PIE.

\subsection{Experimental results}

\subsubsection{Classification rates}

For each dataset, we randomly select $80 \%$ of samples per class for training and the remaining are used for testing. In order to show the reliability of all the methods, the process is repeated for 10 times. After feature selection, we use $L^{2}$-distance based 1-Nearest Neighbor classifier to estimate classification rates. Here MCFS uses the number of classes as its prior parameter because we set the number of eigenvector as the number of classes. Fig.2 plots average classification rates versus the numbers of selected features. Tab.1 shows the average highest classification rates with the corresponding numbers of features obtained by six different methods on two datasets.

From Fig.2 and Tab.1 we can see that Max Variance and Laplacian Score achieve the lowest classification rates than other methods. MCFS and UDFS perform better than Max Variance and Laplacian Score. We can see from Tab.1 that classification rates with all features outperform the highest classification rates of Max Variance and Laplacian Score. Fig.2 shows that on Extended YaleB SPFW is always superior to other feature selection methods under different numbers of features, while on PIE SPFW has an advantage over larger numbers of features. In summary, SPFW achieves the highest classification rates with fewer features.

\subsubsection{Clustering}

We evaluate the clustering performance by Normalized Mutual Information (NMI) [35]. A larger NMI indicates a better clustering result. The numbers of clusters are 


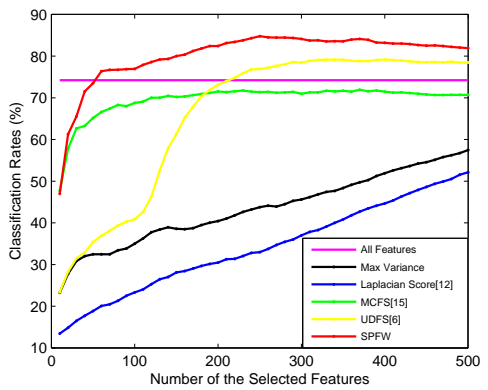

(a) Extended YaleB

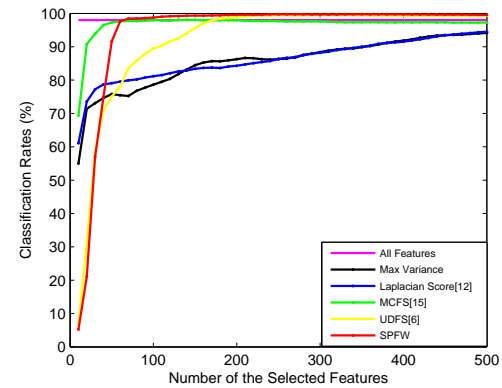

(b)PIE

Figure 2: Classification rates vs. the numbers of selected features on two face datasets: (a) Extended YaleB (b) PIE.

Table 1: Comparison of the average highest classification rates (\%) with corresponding numbers of features obtained by six different methods on two face datasets. The best results are highlighted in bold.

\begin{tabular}{|c|c|c|c|c|c|c|}
\hline Databases & All Features & Max Variance & Laplacian Score [12] & MCFS [15] & UDFS [6] & SPFW \\
\hline \hline \multirow{2}{*}{ Extended YaleB } & 74.20 & 57.47 & 52.14 & 71.92 & 79.20 & $\mathbf{8 4 . 7 6}$ \\
& $(1024)$ & $(500)$ & $(500)$ & $(370)$ & $(400)$ & $(250)$ \\
\hline \multirow{2}{*}{ PIE } & 98.00 & 94.14 & 94.50 & 98.09 & 99.49 & $\mathbf{9 9 . 7 4}$ \\
& $(1024)$ & $(500)$ & $(500)$ & $(140)$ & $(260)$ & $(350)$ \\
\hline
\end{tabular}


Table 2: Comparison of the average highest clustering performance (NMI\%) with std obtained by six different methods on Extended YaleB. The best results are highlighted in bold.

\begin{tabular}{|c|c|c|c|c|c|c|}
\hline Databases & All Features & Max Variance & Laplacian Score [12] & MCFS [15] & UDFS [6] & SPFW \\
\hline \hline 10 Clusters & $5.81 \pm 0.19$ & $6.10 \pm 0.20$ & $7.71 \pm 0.26$ & $10.69 \pm 2.05$ & $7.73 \pm 0.21$ & $\mathbf{1 6 . 7 0} \pm \mathbf{0 . 4 7}$ \\
\hline 20 Clusters & $8.39 \pm 0.47$ & $9.63 \pm 0.50$ & $10.81 \pm 0.16$ & $19.36 \pm 1.82$ & $11.74 \pm 0.90$ & $\mathbf{2 4 . 9 0} \pm \mathbf{0 . 9 1}$ \\
\hline 30 Clusters & $12.24 \pm 0.98$ & $12.87 \pm 0.34$ & $13.71 \pm 0.22$ & $23.99 \pm 2.26$ & $16.25 \pm 0.80$ & $\mathbf{3 0 . 9 4} \pm \mathbf{0 . 9 6}$ \\
\hline 38 Clusters & $15.32 \pm 0.70$ & $15.48 \pm 0.47$ & $16.03 \pm 0.39$ & $27.12 \pm 1.34$ & $19.41 \pm 0.89$ & $\mathbf{3 5 . 9 4} \pm \mathbf{0 . 6 1}$ \\
\hline
\end{tabular}

respectively set as 10, 20, 30, 38 on Extended YaleB and 20,40,60,68 on PIE. As mentioned in [15], MCFS achieves its best performance as the number of used eigenvectors is equal to the number of clusters. Therefore, we set the number of eigenvector as the number of clusters for MCFS. Following Ref.[15], we apply Kmeans algorithm to cluster samples with the selected features. Since the performance of Kmeans clustering depends on initialization, we repeat the clustering 10 times with random initialization. For each dataset, we randomly select $80 \%$ of samples per class for testing and repeat the process 10 times. Fig. 3 and Fig. 4 show the average NMI versus the numbers of selected features on Extended YaleB and PIE respectively. Tab.2 and Tab.3 show the average highest NMI with standard deviation obtained by six different methods on two datasets respectively.

Tab. 2 and Tab. 3 show that, for all methods, the clustering performance increases in general as the number of cluster increases and the performance difference among different methods gets smaller. From Fig. 3 and Fig.4 we can see that MCFS and UDFS are always superior to All Features, Max Variance and Laplacian Score. Fig.3 shows that on Extended YaleB the performance of SPFW with varying numbers of selected features and clusters are always better than that of other methods. In Fig. 4 we observe that on PIE SPFW outperforms other methods when the number of features is more than 30 .

All in all, from the above experimental results we have the following observations. (1) Most feature selection methods perform much better than All Features, which 


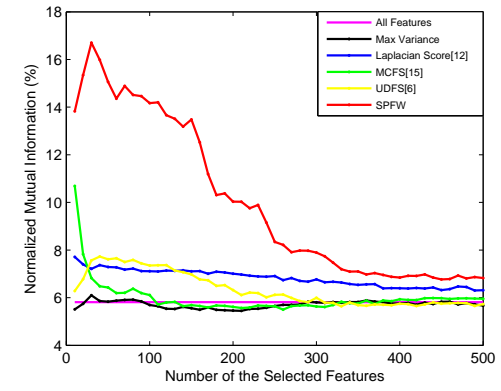

(a) 10 clusters

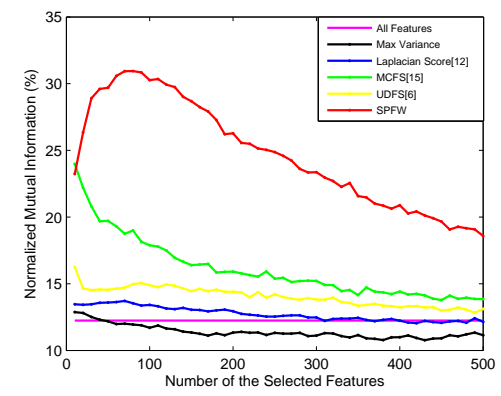

(c) 30 clusters

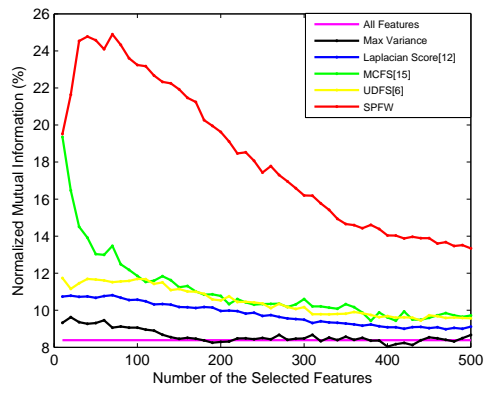

(b) 20 clusters

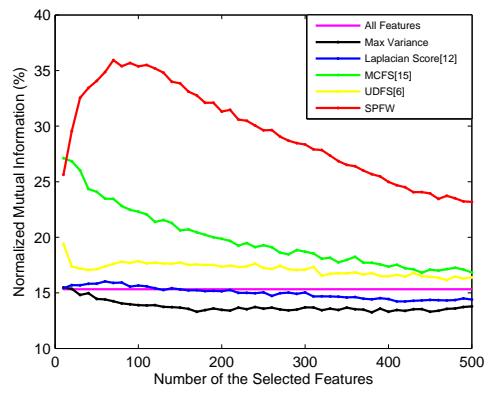

(d) 38 clusters

Figure 3: Clustering performances vs. the numbers of selected features on Extended YaleB with different numbers of clusters.

Table 3: Comparison of the average highest clustering performance (NMI\%) with std obtained by six different methods on PIE. The best results are highlighted in bold.

\begin{tabular}{|c|c|c|c|c|c|c|}
\hline Databases & All Features & Max Variance & Laplacian Score [12] & MCFS [15] & UDFS [6] & SPFW \\
\hline \hline 20 Clusters & $17.76 \pm 0.56$ & $24.29 \pm 0.66$ & $21.87 \pm 0.58$ & $30.62 \pm 2.73$ & $34.63 \pm 0.99$ & $\mathbf{3 9 . 2 1} \pm \mathbf{0 . 8 3}$ \\
\hline 40 Clusters & $30.63 \pm 0.91$ & $34.11 \pm 0.75$ & $32.63 \pm 0.75$ & $41.55 \pm 2.68$ & $46.57 \pm 0.82$ & $\mathbf{5 2 . 3 3} \pm \mathbf{0 . 8 1}$ \\
\hline 60 Clusters & $38.69 \pm 0.72$ & $40.11 \pm 1.05$ & $39.39 \pm 1.67$ & $48.15 \pm 3.19$ & $53.33 \pm 0.90$ & $\mathbf{5 9 . 8 2} \pm \mathbf{0 . 5 8}$ \\
\hline 68 Clusters & $42.44 \pm 0.46$ & $43.32 \pm 0.58$ & $42.86 \pm 1.87$ & $51.30 \pm 3.75$ & $57.07 \pm 0.98$ & $\mathbf{6 4 . 6 2} \pm \mathbf{0 . 6 4}$ \\
\hline
\end{tabular}




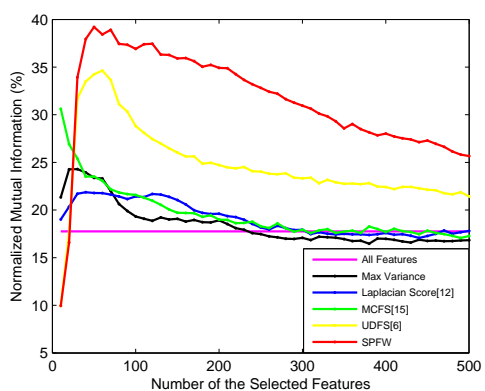

(a) 20 clusters

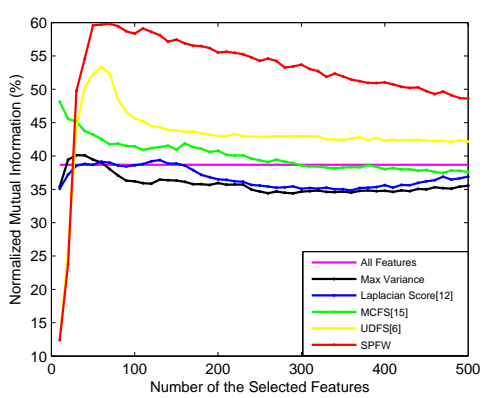

(c) 60 clusters

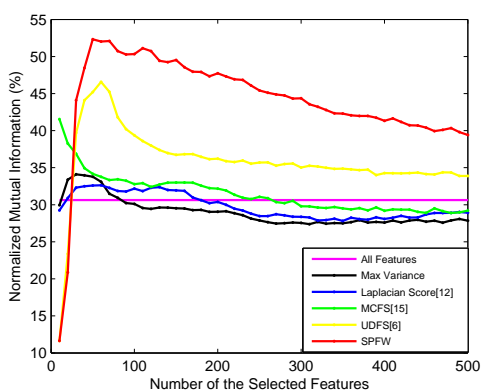

(b) 40 clusters

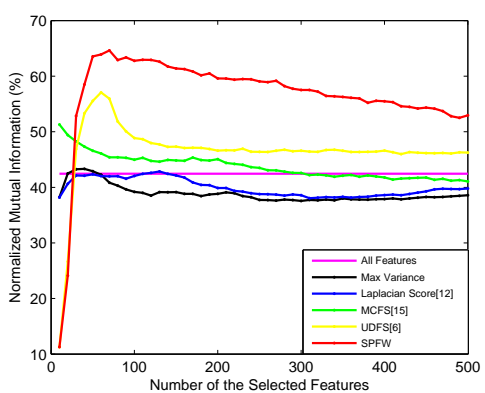

(d) 68 clusters

Figure 4: Clustering performances vs. the numbers of selected features on PIE with different numbers of clusters. 
demonstrates feature selection can improve classification and clustering performance. (2) MCFS and UDFS gain better performance than Max Variance and Laplacian Score. This is because both MCFS and UDFS exploit discriminative information and select features in batch mode. It validates that it is a better way to analyze data features jointly with discriminative information for feature selection. (3) SPFW obtains the best performance in both classification and clustering results. There are three main reasons for this. Firstly, SPFW defines the sparse representation based reconstruction residual as measurement criterion, thus the locality relationship among samples is adaptively preserved. As previous work [27] mentioned, sparse representation has been demonstrated to be very effective for face recognition. Secondly, SPFW incorporates feature weighting to sparse representation, which provides a mechanism for further noise removing. Lastly, SPFW selects features in batch mode, because it learns feature weights jointly rather than individually. (4) On PIE, SPFW has no advantage under smaller numbers of selected features. This is because the sparest solution of $\alpha_{i}$ under $l_{1}$-norm minimization may have no discriminative information when feature dimension is far less than the number of samples.

\subsection{Convergence performance}

In Algorithm 1, we have two separate steps to solve the feature weights vector $w$, and sparse representation coefficients $\left\{\alpha_{i}\right\}$ in a loop. In each iteration, the reduced sub-problems can be efficiently solved with some off-the-shelf toolboxes. The objective function values over different iteration numbers are given in Fig.5. As it can be observed that the objective values become stable after around 10 iterations.

\subsection{Parameter sensitivity}

In our Algorithm 1, $\beta$ should be set in advance. We show the classification performance with respect to the variation of $\beta$ and the number of selected features in Fig.6. As can be seen, the performances are not very sensitive when parameter $\beta$ is within $\left\{10^{-3}, 10^{-2}, 10^{-1}, 1\right\}$. In contrast, our algorithm is sensitive to the number of selected features. And how to identify the number of optimal features remains an open problem. 


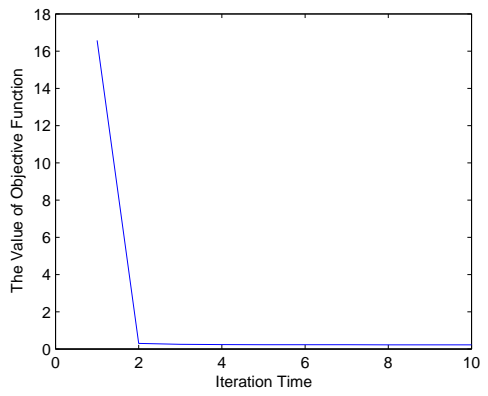

(a) Extended YaleB

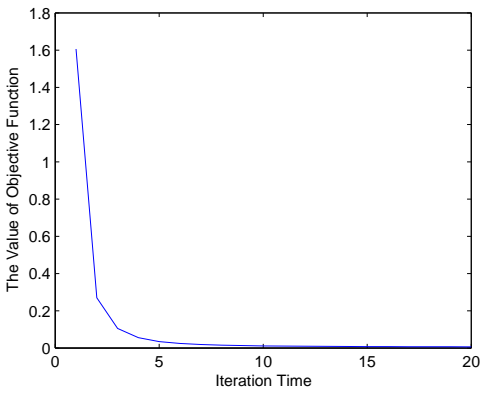

(b) PIE

Figure 5: The convergence curve of the SPFW algorithm on two face datasets: (a) Extended YaleB (b) PIE.

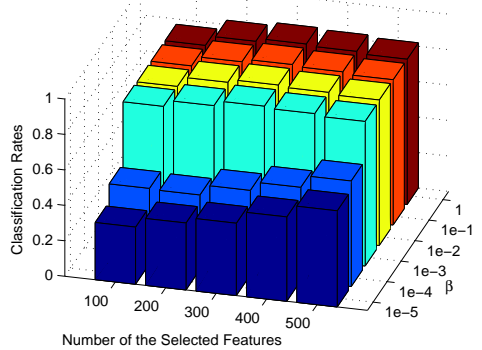

(a) Extended YaleB

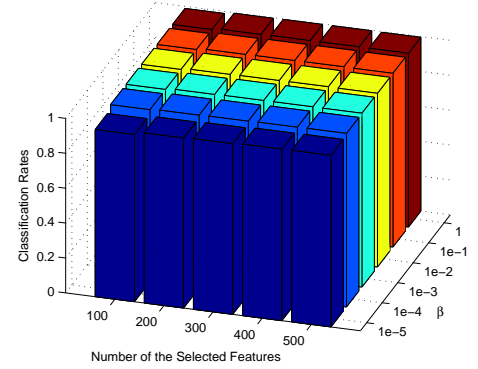

(b) PIE

Figure 6: Classification rates of SPFW with different $\beta$ s and feature numbers on Extended YaleB and PIE. 
Table 4: Comparison of the CPU time(s) for training of different methods on Extended YaleB and PIE.

\begin{tabular}{|c|c|c|c|c|c|}
\hline Databases & Max Variance & Laplacian Score [12] & MCFS [15] & UDFS [6] & SPFW \\
\hline \hline Extended YaleB & 0.017 & 0.401 & 76.6 & 27.9 & 62.7 \\
\hline PIE & 0.021 & 0.476 & 207 & 41.7 & 91.0 \\
\hline
\end{tabular}

\subsection{Computational time}

We perform different feature selection algorithms on a computer with two CPUs and each CPU is $2.6 \mathrm{GHz}$ with 8-core. The computational times are shown in Tab.4. For MCFS, we set the number of selected features as 500 in the training process. We can see from Tab.4 that: (1) Greedy feature selection algorithms such as Max Variance and Laplacian Score run faster than joint feature selection algorithms such as MCFS, UDFS, and SPFW. (2) Being heavily time consuming in computing $l_{1}$-regularized regression problem, MCFS costs the most time in all the cases. UDFS and SPFW have comparable computational time, and run faster than MCFS. SPFW spends much time on computing different $\alpha_{i}$ s, respectively. Since different $\alpha_{i} \mathrm{~s}$ are independent from each other, in our future work we can adopt the parallel computing technology to further accelerate.

\section{Conclusion}

In this paper, we proposed a novel unsupervised feature selection method, SPFW, which took into account the joint feature selection and sparse representation simultaneously. And we compared SPFW with other state-of-the-art unsupervised feature selection methods in terms of classification and clustering. The experimental results on two face image datasets (Extended YaleB and PIE) demonstrated the effectiveness of the proposed SPFW.

\section{Acknowledgments}

This work is supported by the National Science Foundation of China (Grant no. 61202134), National Science Fund for Distinguished Young Scholars (Grant no. 61125305), 
China Postdoctoral Science Foundation, and the Postdoctoral Science Foundation of Jiangsu Province.

[1] R. Pinheiro, G. Cavalcanti, T. Re, Data-driven global-ranking local feature selection methods for text categorization, Expert Systems with Applications 42(4) (2015) 1941-1949.

[2] D. Yu, J. Hu, H. Yan, X. Yang, J. Yang, H. Shen, Enhancing protein-vitamin binding residues prediction by multiple heterogeneous subspace $S V M$ s ensemble, BMC Bioinformatics 15 (2014) 297.

[3] J. Zhang, J. Yang, J. Qian, J. Xu, Nearest orthogonal matrix representation for face recognition, Neurocomputing 151(3) (2015) 471-480.

[4] Z. Zhao, H. Liu, Spectral feature selection for supervised and unsupervised learning, International Conference on Machine Learning. Corvallis, USA (2007) $1151-1157$.

[5] Z. Zhao, L. Wang, H. Liu, J. Ye, On similarity preserving feature selection, IEEE Transactions on Knowledge and Data Engineering 25(3) (2011) 619-632.

[6] Y. Yang, H. Shen, Z. Ma, Z. Huang, X. Zhou, $L_{2,1}$-norm regularized discriminative feature selection for unsupervised learning, International Joint Conference on Artificial Intelligence. Barcelona, Spain (2011) 1589-1594.

[7] R. Duda, P. Hart, D.Stork, Pattern Classification, 2nd Edition, John Wiley \& Sons, 2001.

[8] H. Peng, F. Long, C. Ding, Feature selection based on mutual information: criteria of max-dependency, max-relevance, and min-redundancy, IEEE Transactions on Pattern Analysis and Machine Intelligence 27(8) (2005) 1226-1238.

[9] I. Kononenko, Estimating attributes: analysis and extensions of RELIEF, European Conference on Machine Learning. Catania, Italy (1994) 171-182. 
[10] F. Nie, H. Huang, X. Cai, C. Ding, Efficient and robust feature selection via joint $l_{2,1}$-norms minimization, Advances in Neural Information Processing Systems. Vancouver, Canada (2010) 1-9.

[11] F. Nie, X. Wang, H. Huang, Clustering and projected clustering with adaptive neighbors, International Conference on Knowledge Discovery and Data Mining. New York, USA (2014) 977-986.

[12] X. He, D. Cai, P. Niyogi, Laplacian score for feature selection, Advances in Neural Information Processing Systems. Vancouver, Canada.

[13] Y. R. Su, C. X. Li, R. J. Wang, P. Chen, Sparse representation-based approach for unsupervised feature selection, ,International Journal of Pattern Recognition and Artificial Intelligence 28(3).

[14] Y. Sun, Iterative RELIEF for feature weighting: algorithms, theories, and applications, IEEE Transactions on Pattern Analysis and Machine Intelligence 29(6) (2007) 1035-1051.

[15] D. Cai, C. Zhang, X. He, Unsupervised feature selection for multi-cluster data, ACM Special Interest Group on Knowledge Discovery and Data Mining. Washington, USA (2010) 333-342.

[16] C. P. Hou, F. P. Nie, D. Y. Yi, Y. Wu, Feature selection via joint embedding learning and sparse regression, International Joint Conferences on Artificial Intelligence. Barcelona, Spain (2014) 793-804.

[17] L. Shi, L. Du, Y. D. Shen, Robust spectral learning for unsupervised feature selection, IEEE International Conference on Data Mining. Shenzhen, China (2014) 977-982.

[18] J. Wright, A. Yang, A. Ganesh, S. Sastry, Y. Ma, Robust face recognition via sparse representation, IEEE Transactions on Pattern Analysis and Machine Intelligence 31 (2) (2009) 210-227. 
[19] B. Cheng, J. Yang, S. Yan, Y. Fu, T. Huang, Learning with $l_{1}$-graph for image analysis, IEEE Transactions on Image Processing 19 (4) (2010) 858-866.

[20] D. Donoho, For most large underdetermined systems of linear equations the minimal $l_{1}$-norm solution is also the sparsest solution, Communications on Pure and Applied Mathematics 59 (6) (2006) 797-829.

[21] E. Candes, J. Romberg, T. Tao, Stable signal recovery from incomplete and inaccurate measurements, Communications on Pure and Applied Mathematics 59 (8) (2006) 1207-1223.

[22] S. Chen, D. Donoho, M. Saunders, Atomic decomposition by basis pursuit, Society for Industry and Applied Mathematics Review 43 (1) (2001) 129-159.

[23] D. Donoho, Y. Tsaig, Fast solution of $l_{1}$-norm minimization problems when the solution may be sparse, IEEE Transactions on Information Theory 54 (11) (2008) 4789-4812.

[24] E. Amaldi, V. Kann, On the approximation of minimizing non zero variables or unsatisfied relations in linear systems, Theoretical Computer Science 209 (1998) $237-260$.

[25] S. Liu, H. Liu, L. Latecki, S. Yan, C. Xu, H. Lu, Size adaptive selection of most informative features, AAAI Conference on Artificial Intelligence. San Francisco, USA.

[26] J. Wang, H. Bensmail, X. Gao, Feature selection and multi-kernel learning for sparse representation on a manifold, Neural Networks 51 (2014) 9-16.

[27] J. Yang, D. Chu, L. Zhang, Y. Xu, J. Yang, Sparse representation classifier steered discriminative projection with applications to face recognition, IEEE Transactions on Neural Networks and Learning Systems 24(7) (2013) 1023-1035.

[28] H. Yan, J. Yang, Locality preserving score for joint feature weights learning, Neural Networks 69 (2015) 126-134. 
[29] Y. Xia, C. Sun, A novel neural dynamical approach to convex quadratic program and its efficient applications, Neural Networks 22 (10) (2009) 1463-1470.

[30] M. Fukushima, P. Tseng, An implementable active-set algorithm for computing a b-stationary point of a mathematical program with linear complementarity constraints, SIAM Journal on Optimization 12(3) (2002) 724-739.

[31] A. Ioffe, Composite optimization: second order conditions, value functions and sensityvity, Analysis and Optimization of Systems (1990) 442-451.

[32] K. Lee, J. Ho, D. Kriegman, Acquiring linear subspaces for face recognition under variable lighting, IEEE Transactions on Pattern Analysis and Machine Intelligence 27 (5) (2005) 684-698.

[33] T. Sim, S. Baker, M. Bsat, The CMU pose, illumination, and expression database, IEEE Transactions on Pattern Analysis and Machine Intelligence 25 (12) (2003) $1615-1618$.

[34] D. Cai, X. He, J. Han, Spectral regression for efficient regularized subspace learning, IEEE International Conference on Computer Vision. Rio de Janeiro, Brazil (2007) 1-8.

[35] A. Strehl, J. Ghosh, Cluster ensemblesca knowledge reuse framework for combining multiple partitions, Journal of Machine Learning Research 3 (2002) 583-617. 


\title{
Sparse Preserving Feature Weights Learning
}

\author{
Guangsheng Xia, Hui Yan*, Jian Yang \\ School of Computer Science and Engineering, \\ Nanjing University of Science and Technology, \\ 210094, China
}

\begin{abstract}
In this paper, we propose a novel unsupervised feature selection algorithm, named sparse preserving feature weights learning (SPFW), which is based on the recent local data representation theory, sparse representation. SPFW differs from traditional feature selection algorithms in two aspects: (1) SPFW is designed on the locality measurement criterion with sparse reconstruction residual minimization. It adaptively determines the locality based on sparse representation, instead of fixing the $k$-nearest neighbors in the original feature space. (2) SPFW selects the most discriminative feature subset from the whole feature set in batch mode, instead of selecting features individually. To optimize the proposed formulation, we propose an efficient iterative algorithm, where each iteration reduces to a subproblem which can be solved with some off-the-shelf toolboxes. We conduct experiments on two face datasets to evaluate the performance of feature selection in terms of classification and clustering, which demonstrate the effectiveness of the proposed algorithm.
\end{abstract}

Keywords: Joint feature selection, Sparse representation, Feature weights learning

\section{Introduction}

Data are often represented as high-dimensional feature vectors in many applications, such as text processing [1], gene expression analysis [2], and face recognition [3]. However, features are often correlated or redundant to each other, and they may

*Corresponding author: Tel.: +86 025 84317297-3038.

Email address: yanhui@njust.edu.cn

Preprint submitted to Neurocomputing

January 16, 2015 
even be corrupted by noises. Usually only a small set of features are really important. Consequently, it is necessary to reduce dimensionality, which can be achieved by feature selection or feature extraction. Feature selection seeks the most informative subset of features from original feature set, while feature extraction transforms an original high-dimensional feature space to a low-dimensional space by combining original features. Compared with feature extraction, feature selection improves result comprehensibility.

During recent years, feature selection has drawn increasing attention, and many feature selection algorithms have been proposed. According to the availability of label information, feature selection can be classified into two main families: unsupervised [4], [5], [6] and supervised feature selection algorithms [7], [8], [9], [10]. Supervised feature selection algorithms can mine the correlation between features and data labels that will guide the search for discriminative features. However, in many data mining applications, data labels are absent. Therefore, unsupervised feature selection is indispensable and it is considered to be much harder than supervised scenario. In this paper we focus on unsupervised feature selection.

Unsupervised feature selection algorithms frequently use data similarity preserving as measurement criterion [11] to assess the importance of features. For instance, Laplacian Score [12] first constructs a $k$-nearest neighbor graph. Then it computes scores for each feature according to their abilities of preserving nearest neighbor relationship in the original feature space. Finally it selects the features one by one with the lowest feature scores.

Traditional locality preserving based feature selection algorithms have two widely acknowledged limitations.

1. Similarity is characterized by $k$-nearest neighbor relationship defined in original feature space, which may be inconsistent with the one in the space of selected features [13]. Therefore, the output of feature subset is suboptimal. Moreover, the optimal number of nearest neighbors, i.e., $k$, depends on datasets and so far how to determine the value of $k$ is still not solved.

2. Greedy feature selection algorithms compute importance for each feature inde- 
pendently. The limitation is that the correlation and redundancy among features are neglected.

The sparse regularization model based algorithms have been proposed to address the second problem. Multi-cluster feature selection (MCFS) [14] proposes a two-stage approach, i.e., spectral regression and $l_{1}$-norm minimization, for feature selection. More recently, Nie [10] and Yang [6] apply $l_{2,1}$-norm regularization model to evaluate the importance of the selected features jointly. The $l_{2,1}$-norm minimization term makes feature selection matrix sparse in rows for the sake of feature selection. These efforts confirm that it is more effective to select features jointly. Note that these feature selection matrices are not constrained to be nonnegative. Strictly speaking these algorithms belong to sparse feature extraction, not feature selection.

\subsection{Our contribution}

In this paper, we introduce sparse representation [15], [16] based measurement criterion and joint feature weights learning into a novel unsupervised feature selection method, which is named sparse preserving feature weights learning. Our objective minimizes sparse representation reconstruction residuals in the space of weighted features. Its appealing characteristics are summarized as follows:

- The proposed algorithm selects features with the abilities of preserving local geometric structure. Compared with traditional locality preserving feature selection methods, our proposed algorithm adaptively determines the locality based on sparse representation in the weighted space, instead of fixing the neighboring samples based on pairwise Euclidean distance, which is very sensitive to data noises, in the original space.

- The proposed algorithm selects the optimal feature subset in batch mode by learning a nonnegative constrained feature weight vector, instead of simply adding each feature into the feature pool separately. An effective and efficient iterative algorithm is developed, where each iteration reduces to a subproblem which can be solved with some off-the-shelf toolboxes. 
The remainder of this paper is organized as follows. In Section 2, we briefly review sparse representation. We present our sparse preserving feature weights learning in Section 3. The experiments on two face datasets are completed in Section 4. Finally, we draw a conclusion in Section 5.

\section{Sparse representation}

Let $A=\left[a_{1}, \ldots, a_{n}\right] \in R^{m \times n}$ be the matrix composed of $n$ training samples with $C$ classes, where $m$ is the number of features. Given a test sample $y \in R^{m}$ from the $c$-th class, intuitively $y$ could be well approximated by the linear combination of the training samples from the $c$-th class. Based on this assumption, sparse representation aims to represent a given test sample as a sparse linear combination of all training samples, and the nonzero representation coefficients should concentrate on the training samples with the same class as the test sample.

Mathematically we represent $y$ in an overcomplete dictionary whose basis vectors are training sample themselves, i.e.,

$$
y=A \alpha
$$

where $\alpha \in R^{n}$ is the representation coefficient vector.

Under the assumption, $m<n$, the linear equation (1) is underdetermined, and this representation is naturally sparse. The sparsest solution of $\alpha$ can be sought by the following $l_{1}$-norm minimization problem [17], [18] :

$$
\hat{\alpha}=\arg \min \|\alpha\|_{1}, \text { s.t., } y=A \alpha
$$

where $\|\cdot\|_{1}$ denotes the $l_{1}$-norm. This problem (2) can be solved in polynomial time by standard linear programming algorithms [19] or recently proposed homotopy algorithm [20] .

\section{Sparse preserving feature weights learning}

In this section we first present sparse preserving feature weights learning (SPFW) algorithm for unsupervised feature selection, and then put forward an iterative algorithm to solve the proposed objective function. 


\subsection{Basic idea and formulation}

Feature subset selection can be viewed as a binary optimization problem, and this combinatorial search is often NP hard. To some extent we alleviate this computational issue by using a joint feature weights learning strategy [21], [22]. Let $X=$ $\left[x_{1}, \ldots, x_{n}\right] \in R^{d \times n}$ be the training data matrix in the original feature space. In the weighted feature space, parameterized by a nonnegative vector $w=\left[w_{1}, \ldots, w_{d}\right]^{T} \in$ $R_{+}^{d}$, the feature vector of the $i$-th sample weighted by $w$ can be denoted as $\operatorname{diag}(w) x_{i}$, where $\operatorname{diag}(w) \in R^{d \times d}$ is a diagonal matrix with $w$ on the diagonal.

According to the sparse preserving decision rule [23], smaller sparse reconstruction residuals will lead to better classification results in general. Therefore, we choose to minimize the sparse reconstruction residuals in the weighted feature space as follows:

$$
\begin{aligned}
\min _{w,\left\{\alpha_{i}, i=1, \ldots, n\right\}} & \sum_{i=1}^{n}\left\|\operatorname{diag}(w)\left(x_{i}-X_{i} \alpha_{i}\right)\right\|_{F}^{2}+\beta\left\|\alpha_{i}\right\|_{1} \\
\text { s.t., } & \sum_{j=1}^{d} w_{j}=1, w_{j} \geq 0
\end{aligned}
$$

where $X_{i}=\left[x_{1}, \ldots, x_{i-1}, 0, x_{i+1}, \ldots, x_{n}\right] \in R^{d \times n}$ whose $i$-th column is all-zero vector, $\alpha_{i}$ is the sparse representation coefficient vector for the $i$-th training sample. $X_{i} \alpha_{i}$ is the sparse reconstruction of $x_{i}$ with the rest training samples.

\subsection{Optimization}

Since in the objective function (3), $\left\{\alpha_{i}\right\}$ and $w$ are dependent on each other, it can not have a closed-form solution. Consequently, we update the variables alternately with others fixed by iteratively solving Eq.(3).

\subsubsection{Initialize $\left\{\alpha_{i}\right\}$}

A good initialization of $\left\{\alpha_{i}\right\}$ can speed up the convergence of the optimization and lead to a robust solution. For each training sample $x_{i}(i=1, \ldots, n)$ in the original feature space, we represent it using the remaining training samples. Its corresponding sparse representation coefficient vector $\alpha_{i}$ is calculated by solving the $l_{1}$-norm optimization problem.

$$
\alpha_{i}=\arg \min \left\|\alpha_{i}\right\|_{1} \text {, s.t., } x_{i}=X_{i} \alpha_{i}
$$




\subsubsection{Update $w$ as given $\left\{\alpha_{i}\right\}$}

By fixing $\left\{\alpha_{i}\right\}$ and removing the irrelevant terms in Eq.(3), we get the following optimization problem with respect to $w$ :

$$
\begin{array}{ll}
\min _{w} & \sum_{i=1}^{n}\left\|\operatorname{diag}(w)\left(x_{i}-X_{i} \alpha_{i}\right)\right\|_{F}^{2} \\
\text { s.t., } & \sum_{j=1}^{d} w_{j}=1, w_{j} \geq 0
\end{array}
$$

Let $\alpha^{w}=\left[\alpha_{1}, \ldots, \alpha_{n}\right]$ and $Y^{w}=X-X \alpha^{w}$, the objective function in the above optimization problem can be represented in the following matrix form:

$$
\begin{aligned}
& \sum_{i=1}^{n}\left\|\operatorname{diag}(w)\left(x_{i}-X_{i} \alpha_{i}\right)\right\|_{F}^{2} \\
= & \left\|\operatorname{diag}(w)\left(X-X \alpha^{w}\right)\right\|_{F}^{2} \\
= & \operatorname{Tr}\left(\operatorname{diag}^{2}(w) Y^{w} Y^{w T}\right)
\end{aligned}
$$

Finally, Eq.(5) is convert to the following problem:

$$
\begin{array}{ll}
\min & \sum_{j=1}^{d} w_{j}^{2}\left(\sum_{i=1}^{n} Y_{j i}^{w^{2}}\right) \\
\text { s.t., } & \sum_{j=1}^{d} w_{j}=1, w_{j} \geq 0
\end{array}
$$

This is a typical quadratic programming [24], which can be solved by the active set algorithm [25].

\subsubsection{Update $\left\{\alpha_{i}\right\}$ as given $w$}

By fixing $w$ and removing the irrelevant terms in Eq.(3), we get the following optimization problem with respect to all $\alpha_{i}$ s:

$$
\min _{\left\{\alpha_{i}\right\}} \sum_{i=1}^{n}\left\|\operatorname{diag}(w)\left(x_{i}-X_{i} \alpha_{i}\right)\right\|_{F}^{2}+\beta\left\|\alpha_{i}\right\|_{1}
$$

Since Eq.(8) is independent among different $i$, we can solve the following problem alternately for each $\alpha_{i}$ :

$$
\min _{\alpha_{i}}\left\|\operatorname{diag}(w)\left(x_{i}-X_{i} \alpha_{i}\right)\right\|_{F}^{2}+\beta\left\|\alpha_{i}\right\|_{1}
$$


Denote $L\left(\alpha_{i}\right)=\left\|\operatorname{diag}(w)\left(x_{i}-X_{i} \alpha_{i}\right)\right\|_{F}^{2}$. In Eq.(9), the first term $L\left(\alpha_{i}\right)$ is smooth, while in the second term $\left\|\alpha_{i}\right\|_{1}$ is non-smooth. $\beta$ is the regularization parameter. Then, we aim to minimize Eq.(9) with a two-step strategy [22] in each iteration:

- Step 1: Update $\hat{\alpha_{i}}$ via the following formulation:

$$
\hat{\alpha_{i}}=\arg \min _{\alpha_{i}}\left\|\operatorname{diag}(w)\left(x_{i}-X_{i} \alpha_{i}\right)\right\|_{F}^{2}
$$

The solution of (10) is given via subgradient descent as:

$$
\hat{\alpha_{i}}=\alpha_{i}-\lambda \frac{d\left(L\left(\alpha_{i}\right)\right)}{d\left(\alpha_{i}\right)}
$$

where $\lambda$ is the step size and set to be $\frac{1}{t} \cdot \frac{d\left(L\left(\alpha_{i}\right)\right)}{d\left(\alpha_{i}\right)}=2\left(X_{i}^{w}\right)^{T}\left(-x_{i}^{w}+X_{i}^{w} \alpha_{i}\right)$, where $X_{i}^{w}=\operatorname{diag}(w) X_{i}$, and $x_{i}^{w}=\operatorname{diag}(w) x_{i}$.

- Step 2: Update $\alpha_{i}$, which is the approximation of $\hat{\alpha_{i}}$ under the $l_{1}$-norm minimization constraint.

$$
\alpha_{i}=\arg \min _{\alpha_{i}} \frac{1}{2}\left\|\alpha_{i}-\hat{\alpha}_{i}\right\|_{F}^{2}+\beta \lambda\left\|\alpha_{i}\right\|_{1}
$$

The solution of Eq.(12) is given by [26]

$$
\alpha_{i}=\left(\left|\hat{\alpha_{i}}\right|-\beta \lambda\right)_{+} \bigotimes \operatorname{sgn}\left(\hat{\alpha_{i}}\right)
$$

where $(a)_{+}=\max (0, a), \operatorname{sgn}(a)$ returns the sign of $a$, and $\otimes$ is the Hadamard operator. Note that any element in $\alpha_{i}$ less than $\beta \lambda$ will be set as zero, and this leads to a sparse solution for $\alpha_{i}$. The complete proposed algorithm is described in Algorithm 1. 
Input: Data $X=\left[x_{1}, . ., x_{n}\right] \in R^{d \times n}$, the maximum iteration number $T$, and the regularization parameter $\beta$

Initial: Compute $\alpha_{i}^{0}$ via Eq.(4)

1. for $t=1, \ldots, T$ do

2. Update the feature weights vector $w^{t}$ by fixing $\alpha_{i}^{t-1}$ via Eq.(7)

3. for $i=1, \ldots, n$ do

4. $\quad$ Update the $i$-th sparse representation $\alpha_{i}^{t}$ via Eq.(11) and Eq.(13)

by fixing $w^{t}$ and other $\alpha_{j}^{t}(j \neq i)$

5. end for

6. end for

Output: $w^{t}$ and $\left\{\alpha_{i}^{t}\right\}$

Algorithm 1: Sparse preserving feature weights learning (SPFW)

\section{Experiments and analysis}

In this section, we first test the performance of the proposed algorithm with some state-of-the-art methods for unsupervised feature selection in terms of classification and clustering. And then we study the convergence performance of SPFW.

\subsection{Experimental data and setting}

We compare the proposed algorithm with some state-of-the-art unsupervised feature selection algorithms, including Max Variance, Laplacian Score, MCFS, unsupervised discriminative feature selection (UDFS) [6], and All Features as a baseline method.

The experiments are conducted on two publicly available face image datasets, i.e., Extended YaleB dataset [27] and PIE dataset [28]. Extended YaleB dataset contains 38 subjects under 9 poses and 64 illumination conditions. We use a subset of Extended YaleB dataset in our experiments ${ }^{1}$, which has around 64 near frontal images under different illuminations per subject. PIE dataset contains 68 subjects with over 40000

\footnotetext{
${ }^{1}$ http://www.cad.zju.edu.cn/home/dengcai/Data/FaceData.html
} 


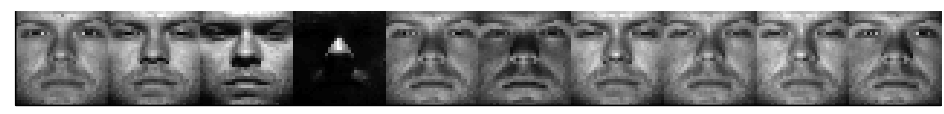

(a) Extended YaleB

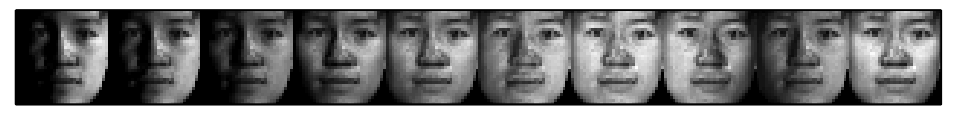

(b) PIE

Figure 1: Samples of cropped images of one person: (a) Extended YaleB (b) PIE.

\subsubsection{Classification rates}

For each dataset, we randomly select $80 \%$ of samples per class for training and the remaining are used for testing. In order to show the reliability of all the methods, the process is repeated for 10 times. After feature selection, we use $L^{2}$-distance based 1-Nearest Neighbor classifier to estimate classification rates. Here MCFS uses the number of classes as its prior parameter because we set the number of eigenvector as the number of classes. Fig.2 plots average classification rates versus the numbers of selected features. Tab.1 shows the average highest classification rates with the corresponding numbers of features obtained by six different methods on two datasets. 


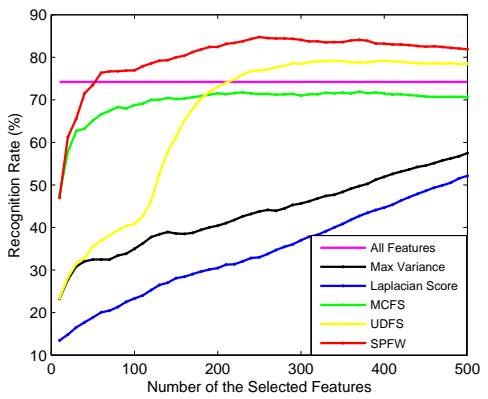

(a) Extended YaleB

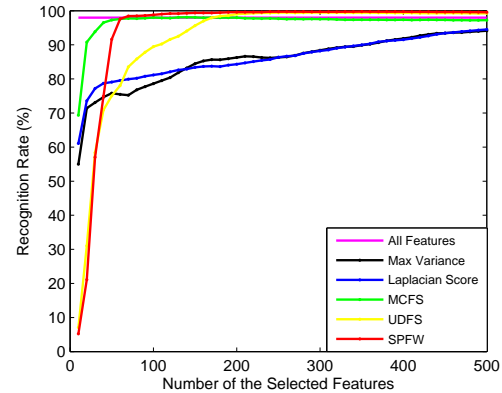

(b)PIE

Figure 2: Classification rates vs. the numbers of selected features on two face datasets: (a) Extended YaleB (b) PIE.

Table 1: Comparison of the average highest classification rates (\%) with corresponding numbers of features obtained by six different methods on two face datasets. The best results are highlighted in bold.

\begin{tabular}{|c|c|c|c|c|c|c|}
\hline Datebases & All Features & Max Variance & Laplacian Score & MCFS & UDFS & SPFW \\
\hline \hline \multirow{2}{*}{ Extended YaleB } & 74.20 & 57.47 & 52.14 & 71.92 & 79.20 & $\mathbf{8 4 . 7 6}$ \\
& $(1024)$ & $(500)$ & $(500)$ & $(370)$ & $(400)$ & $(250)$ \\
\hline \multirow{2}{*}{ PIE } & 98.00 & 94.14 & 94.50 & 98.09 & 99.49 & $\mathbf{9 9 . 7 4}$ \\
& $(1024)$ & $(500)$ & $(500)$ & $(140)$ & $(260)$ & $(350)$ \\
\hline
\end{tabular}
the lowest classification rates than other methods. MCFS and UDFS perform better than Max Variance and Laplacian Score. We can see from Tab.1 that classification rates with all features outperform the highest classification rates of Max Variance and Laplacian Score. Fig.2 shows that on Extended YaleB SPFW is always superior to other feature selection methods under different numbers of features, while on PIE SPFW has an advantage over larger numbers of features. In summary, SPFW achieves the highest classification rates with fewer features. 


\subsubsection{Clustering}

We evaluate the clustering performance by Normalized Mutual Information (NMI) [30]. A larger NMI indicates a better clustering result. The numbers of clusters are respectively set as 10, 20, 30, 38 on Extended YaleB and 20,40,60,68 on PIE. As mentioned in [14], MCFS achieves its best performance as the number of used eigenvectors is equal to the number of clusters. Therefore, we set the number of eigenvector as the number of clusters for MCFS. Following Ref.[14], we apply Kmeans algorithm to cluster samples with the selected features. Since the performance of Kmeans clustering depends on initialization, we repeat the clustering 10 times with random initialization. For each dataset, we randomly select $80 \%$ of samples per class for testing and repeat the process 10 times. Fig. 3 and Fig.4 show the average NMI versus the numbers of selected features on Extended YaleB and PIE respectively. Tab.2 and Tab.3 show the average highest NMI with standard deviation obtained by six different methods on two datasets respectively.

Tab.2 and Tab.3 show that, for all methods, the clustering performance increases in general as the number of cluster increases and the performance difference among different methods gets smaller. From Fig. 3 and Fig.4 we can see that MCFS and UDFS are always superior to All Features, Max Variance and Laplacian Score. Fig.3 shows that on Extended YaleB the performance of SPFW with varying numbers of selected features and clusters are always better than that of other methods. In Fig.4 we observe that on PIE SPFW outperforms other methods when the number of features is more than 30 .

All in all, from the above experimental results we have the following observations. (1) Most feature selection methods perform much better than All Features, which demonstrates feature selection can improve classification and clustering performance. (2) MCFS and UDFS gain better performance than Max Variance and Laplacian Score. This is because both MCFS and UDFS exploit discriminative information and select features in batch mode. It validates that it is a better way to analyze data features jointly with discriminative information for feature selection. (3) SPFW obtains the best performance in both classification and clustering results. There are two main reasons for 


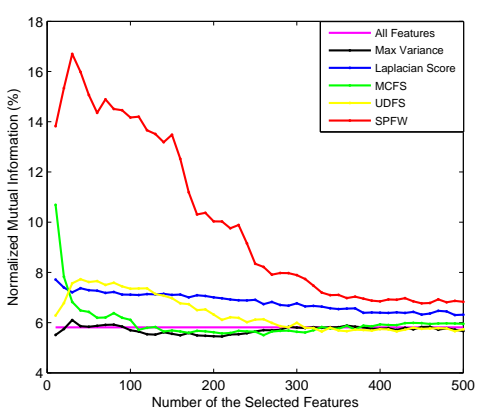

(a) 10 clusters

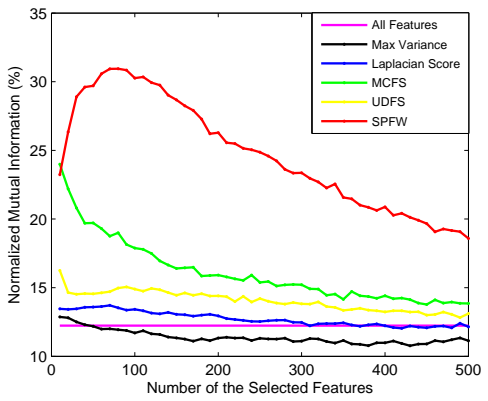

(c) 30 clusters

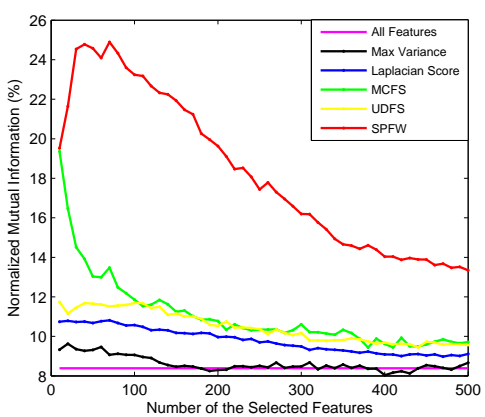

(b) 20 clusters

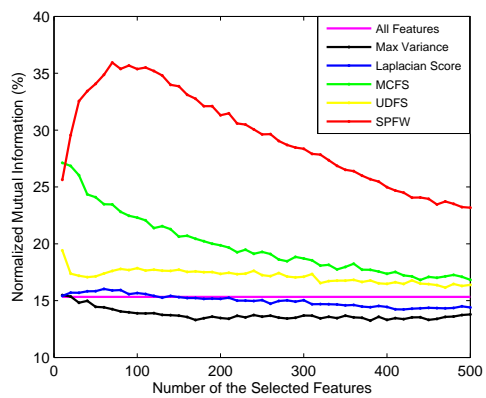

(d) 38 clusters

Figure 3: Clustering performances vs. the numbers of selected features on Extended YaleB with different numbers of clusters.

Table 2: Comparison of the average highest clustering performance (NMI\%) with std obtained by six different methods on Extended YaleB. The best results are highlighted in bold.

\begin{tabular}{|c|c|c|c|c|c|c|}
\hline Datebases & All Features & Max Variance & Laplacian Score & MCFS & UDFS & SPFW \\
\hline \hline 10 Clusters & $5.81 \pm 0.19$ & $6.10 \pm 0.20$ & $7.71 \pm 0.26$ & $10.69 \pm 2.05$ & $7.73 \pm 0.21$ & $\mathbf{1 6 . 7 0} \pm \mathbf{0 . 4 7}$ \\
\hline 20 Clusters & $8.39 \pm 0.47$ & $9.63 \pm 0.50$ & $10.81 \pm 0.16$ & $19.36 \pm 1.82$ & $11.74 \pm 0.90$ & $\mathbf{2 4 . 9 0} \pm \mathbf{0 . 9 1}$ \\
\hline 30 Clusters & $12.24 \pm 0.98$ & $12.87 \pm 0.34$ & $13.71 \pm 0.22$ & $23.99 \pm 2.26$ & $16.25 \pm 0.80$ & $\mathbf{3 0 . 9 4} \pm \mathbf{0 . 9 6}$ \\
\hline 38 Clusters & $15.32 \pm 0.70$ & $15.48 \pm 0.47$ & $16.03 \pm 0.39$ & $27.12 \pm 1.34$ & $19.41 \pm 0.89$ & $\mathbf{3 5 . 9 4} \pm \mathbf{0 . 6 1}$ \\
\hline
\end{tabular}




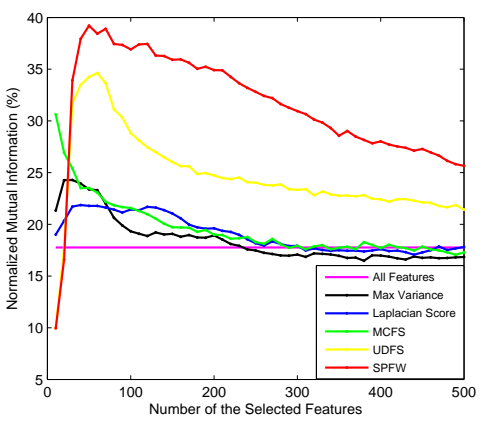

(a) 20 clusters

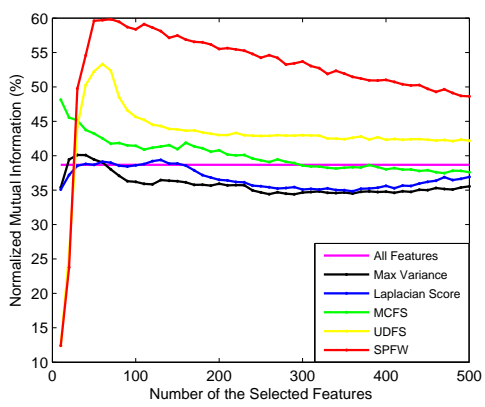

(c) 60 clusters

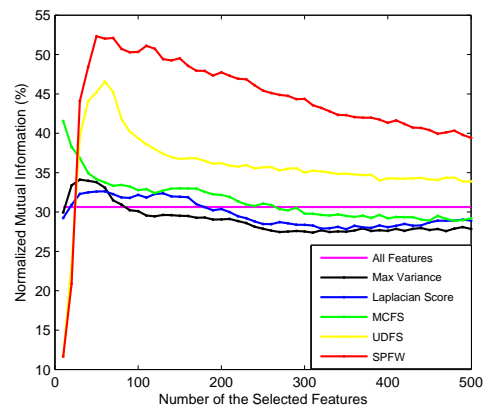

(b) 40 clusters

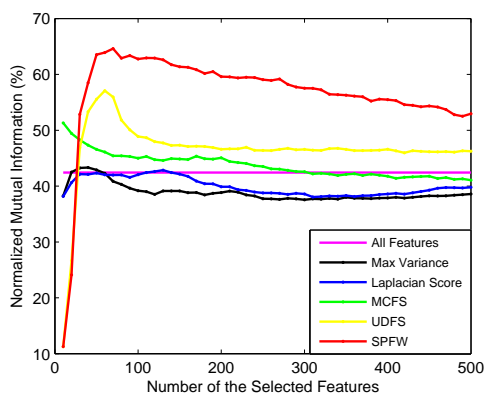

(d) 68 clusters

Figure 4: Clustering performances vs. the numbers of selected features on PIE with different numbers of clusters.

Table 3: Comparison of the average highest clustering performance (NMI\%) with std obtained by six different methods on PIE. The best results are highlighted in bold.

\begin{tabular}{|c|c|c|c|c|c|c|}
\hline Datebases & All Features & Max Variance & Laplacian Score & MCFS & UDFS & SPFW \\
\hline \hline 20 Clusters & $17.76 \pm 0.56$ & $24.29 \pm 0.66$ & $21.87 \pm 0.58$ & $30.62 \pm 2.73$ & $34.63 \pm 0.99$ & $\mathbf{3 9 . 2 1} \pm \mathbf{0 . 8 3}$ \\
\hline 40 Clusters & $30.63 \pm 0.91$ & $34.11 \pm 0.75$ & $32.63 \pm 0.75$ & $41.55 \pm 2.68$ & $46.57 \pm 0.82$ & $\mathbf{5 2 . 3 3} \pm \mathbf{0 . 8 1}$ \\
\hline 60 Clusters & $38.69 \pm 0.72$ & $40.11 \pm 1.05$ & $39.39 \pm 1.67$ & $48.15 \pm 3.19$ & $53.33 \pm 0.90$ & $\mathbf{5 9 . 8 2} \pm \mathbf{0 . 5 8}$ \\
\hline 68 Clusters & $42.44 \pm 0.46$ & $43.32 \pm 0.58$ & $42.86 \pm 1.87$ & $51.30 \pm 3.75$ & $57.07 \pm 0.98$ & $\mathbf{6 4 . 6 2} \pm \mathbf{0 . 6 4}$ \\
\hline
\end{tabular}




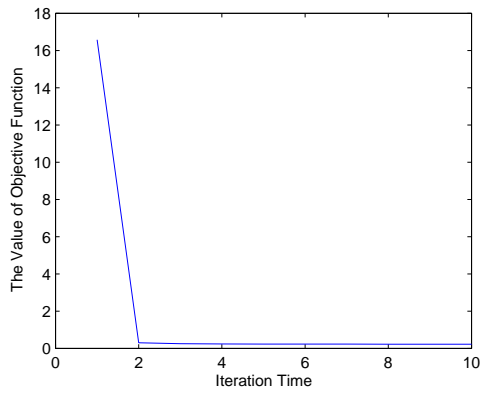

(a) Extended YaleB

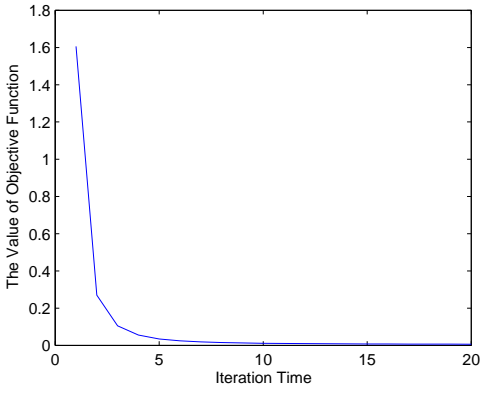

(b) PIE

Figure 5: The convergence curve of the SPFW algorithm on two face datasets: (a) Extended YaleB (b) PIE.

this. First, SPFW defines the sparse representation based reconstruction residual as measurement criterion, thus the locality relationship among samples is adaptively preserved. Second, SPFW selects features jointly rather than individually. (4) On PIE, SPFW has no advantage under smaller numbers of selected features. This is because the sparest solution of $\alpha_{i}$ under $l_{1}$-norm minimization may have no discriminative information when feature dimension is far less than the number of samples.

\subsection{Convergence performance}

In Algorithm 1, we have two separate steps to solve the feature weights vector $w$, and sparse representation coefficients $\left\{\alpha_{i}\right\}$ in a loop. In each iteration, the reduced sub-problems can be efficiently solved with some off-the-shelf toolboxes. The objective function values over different iteration numbers are given in Fig.5. As it can be observed that the objective values become stable after around 10 iterations.

\section{Conclusion}

In this paper, we proposed a novel unsupervised feature selection method, SPFW, which took into account the joint feature selection and sparse representation simultaneously. And we compared SPFW with other state-of-the-art unsupervised feature selection methods in terms of classification and clustering. The experimental results 
on two face image datasets (Extended YaleB and PIE) demonstrated the effectiveness of the proposed SPFW.

\section{Acknowledgments}

This work is supported by the National Science Foundation of China (Grant no. 61202134), National Science Fund for Distinguished Young Scholars (Grant no. 61125305), Jiangsu Province.

\section{References}

[1] R. Pinheiro, G. Cavalcanti, T. Re, Data-driven global-ranking local feature selection methods for text categorization, Expert Systems with Applications 42(4) (2015) 1941-1949.

[2] D. Yu, J. Hu, H. Yan, X. Yang, J. Yang, H. Shen, Enhancing protein-vitamin binding residues prediction by multiple heterogeneous subspace $S V M$ s ensemble, BMC Bioinformatics 15 (2014) 297.

[3] J. Zhang, J. Yang, J. Qian, J. Xu, Nearest orthogonal matrix representation for face recognition, Neurocomputing 151(3) (2015) 471-480.

[4] Z. Zhao, H. Liu, Spectral feature selection for supervised and unsupervised learning, International Conference on Machine Learning. Corvallis, USA (2007) $1151-1157$.

[5] Z. Zhao, L. Wang, H. Liu, J. Ye, On similarity preserving feature selection, IEEE Transactions on Knowledge and Data Engineering 25(3) (2011) 619-632.

[6] Y. Yang, H. Shen, Z. Ma, Z. Huang, X. Zhou, $L_{2,1}$-norm regularized discriminative feature selection for unsupervised learning, International Joint Conference on Artificial Intelligence. Barcelona, Spain (2011) 1589-1594.

[7] R. Duda, P. Hart, D.Stork, Pattern Classification, 2nd Edition, John Wiley \& Sons, 2001. 
[8] H. Peng, F. Long, C. Ding, Feature selection based on mutual information: criteria of max-dependency, max-relevance, and min-redundancy, IEEE Transactions on Pattern Analysis and Machine Intelligence 27(8) (2005) 1226-1238.

[9] I. Kononenko, Estimating attributes: analysis and extensions of RELIEF, European Conference on Machine Learning. Catania, Italy (1994) 171-182.

[10] F. Nie, H. Huang, X. Cai, C. Ding, Efficient and robust feature selection via joint $l_{2,1}$-norms minimization, Advances in Neural Information Processing Systems. Vancouver, Canada (2010) 1-9.

[11] F. Nie, X. Wang, H. Huang, Clustering and projected clustering with adaptive neighbors, International Conference on Knowledge Discovery and Data Mining. New York, USA (2014) 977-986.

[12] X. He, D. Cai, P. Niyogi, Laplacian score for feature selection, Advances in Neural Information Processing Systems. Vancouver, Canada.

[13] Y. Sun, Iterative RELIEF for feature weighting: algorithms, theories, and applications, IEEE Transactions on Pattern Analysis and Machine Intelligence 29(6) (2007) 1035-1051.

[14] D. Cai, C. Zhang, X. He, Unsupervised feature selection for multi-cluster data, ACM Special Interest Group on Knowledge Discovery and Data Mining. Washington, USA (2010) 333-342.

[15] J. Wright, A. Yang, A. Ganesh, S. Sastry, Y. Ma, Robust face recognition via sparse representation, IEEE Transactions on Pattern Analysis and Machine Intelligence 31 (2) (2009) 210-227.

[16] B. Cheng, J. Yang, S. Yan, Y. Fu, T. Huang, Learning with $l_{1}$-graph for image analysis, IEEE Transactions on Image Processing 19 (4) (2010) 858-866.

[17] D. Donoho, For most large underdetermined systems of linear equations the minimal $l_{1}$-norm solution is also the sparsest solution, Communications on Pure and Applied Mathematics 59 (6) (2006) 797-829. 
[18] E. Candes, J. Romberg, T. Tao, Stable signal recovery from incomplete and inaccurate measurements, Communications on Pure and Applied Mathematics 59 (8) (2006) 1207-1223.

[19] S. Chen, D. Donoho, M. Saunders, Atomic decomposition by basis pursuit, Society for Industry and Applied Mathematics Review 43 (1) (2001) 129-159.

[20] D. Donoho, Y. Tsaig, Fast solution of $l_{1}$-norm minimization problems when the solution may be sparse, IEEE Transactions on Information Theory 54 (11) (2008) $4789-4812$.

[21] S. Liu, H. Liu, L. Latecki, S. Yan, C. Xu, H. Lu, Size adaptive selection of most informative features, AAAI Conference on Artificial Intelligence. San Francisco, USA.

[22] J. Wang, H. Bensmail, X. Gao, Feature selection and multi-kernel learning for sparse representation on a manifold, Neural Networks 51 (2014) 9-16.

[23] J. Yang, D. Chu, L. Zhang, Y. Xu, J. Yang, Sparse representation classifier steered discriminative projection with applications to face recognition, IEEE Transactions on Neural Networks and Learning Systems 24(7) (2013) 1023-1035.

[24] Y. Xia, C. Sun, A novel neural dynamical approach to convex quadratic program and its efficient applications, Neural Networks 22 (10) (2009) 1463-1470.

[25] M. Fukushima, P. Tseng, An implementable active-set algorithm for computing a b-stationary point of a mathematical program with linear complementarity constraints, SIAM Journal on Optimization 12(3) (2002) 724-739.

[26] A. Ioffe, Composite optimization: second order conditions, value functions and sensityvity, Analysis and Optimization of Systems (1990) 442-451.

[27] K. Lee, J. Ho, D. Kriegman, Acquiring linear subspaces for face recognition under variable lighting, IEEE Transactions on Pattern Analysis and Machine Intelligence 27 (5) (2005) 684-698. 
[28] T. Sim, S. Baker, M. Bsat, The CMU pose, illumination, and expression database, IEEE Transactions on Pattern Analysis and Machine Intelligence 25 (12) (2003) 1615-1618.

[29] D. Cai, X. He, J. Han, Spectral regression for efficient regularized subspace learning, IEEE International Conference on Computer Vision. Rio de Janeiro, Brazil (2007) 1-8.

300 [30] A. Strehl, J. Ghosh, Cluster ensemblesca knowledge reuse framework for combining multiple partitions, Journal of Machine Learning Research 3 (2002) 583-617. 
Guangsheng Xia received her B.S. degree in Network Engineering from Hebei Normal University(HEBNU), Shijiazhuang, China, in 2013. Now, she is a candidate of the master degree in Software Engineering from the School of Computer Science and Technology, Nanjing University of Science and Technology (NUST), Nanjing, China. Her research interests include pattern recognition and machine learning. 
Hui Yan received her B.S. degree and Ph.D. degree from the School of Computer Science and Technology, Nanjing University of Science and Technology (NUST), Nanjing, China, in 2005 and 2011, respectively. In 2009, she was a visiting student at the Department of Electrical and Computer Engineering at National University of Singapore, Singapore. She is currently a lecturer at the School of Computer Science and Engineering, NUST. Her research interests include pattern recognition, computer vision and machine learning. 
Jian Yang received his B.S.degree in mathematics from Xuzhou Normal University, Xuzhou, China, in 1995, his M.S.degree in applied mathematics from Changsha Railway University, Changsha, China, in1998, and his Ph.D. degree in pattern recognition and intelligence systems from Nanjing University of Science and Technology (NUST), Nanjing, China, in 2002. He was a Post-Doctoral Researcher at the University of Zaragoza, Spain, in 2003. From 2004 to 2006, he was a Post-Doctoral Fellow at the Biometrics Centre of Hong Kong Polytechnic University, Hong Kong. From 2006 to 2007, at the Department of Computer Science, New Jersey Institute of Technology, Newark, NJ, USA. He is currently a Professor at the School of Computer Science and Technology, NUST. He has authored more than 80 academic papers in pattern recognition and computer vision. His journal papers have been cited more than 1800 times in the ISI Web of Science, and 3000 times in the Web of Scholar Google. His current research interests include pattern recognition, computer vision and machine learning. 
${ }^{*}$ Photo of the author(s)

Click here to download high resolution image

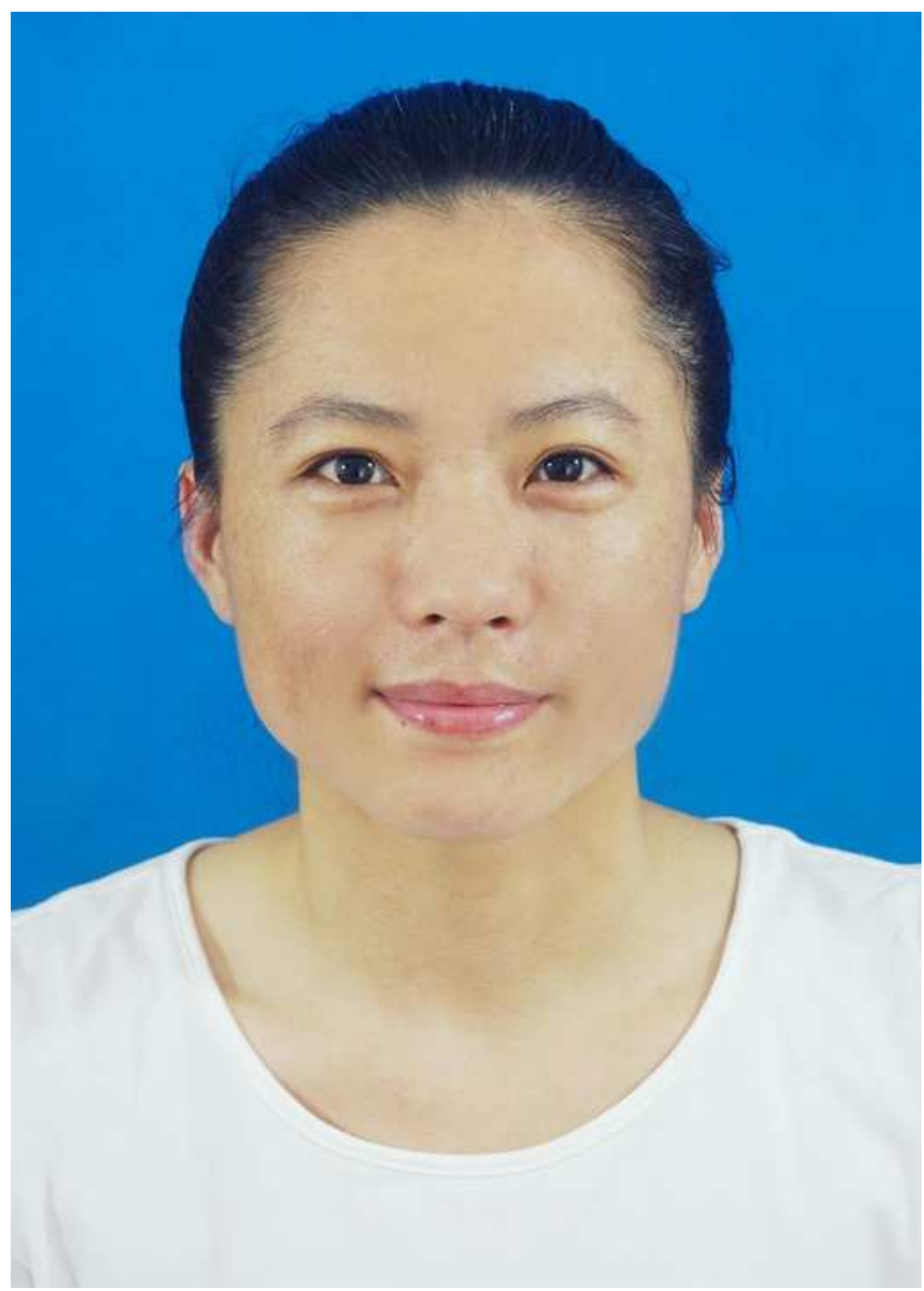


${ }^{*}$ Photo of the author(s)

Click here to download high resolution image

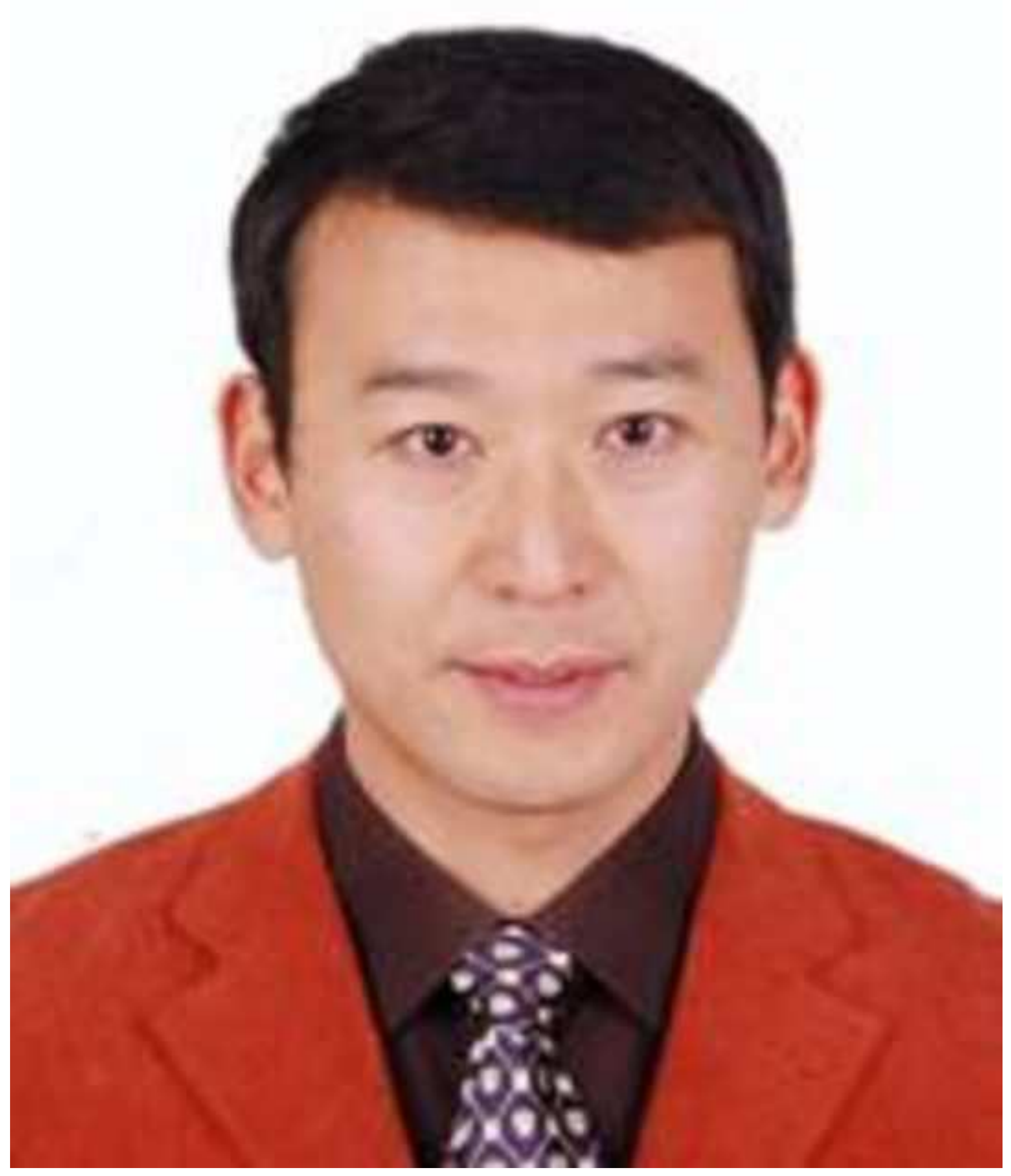




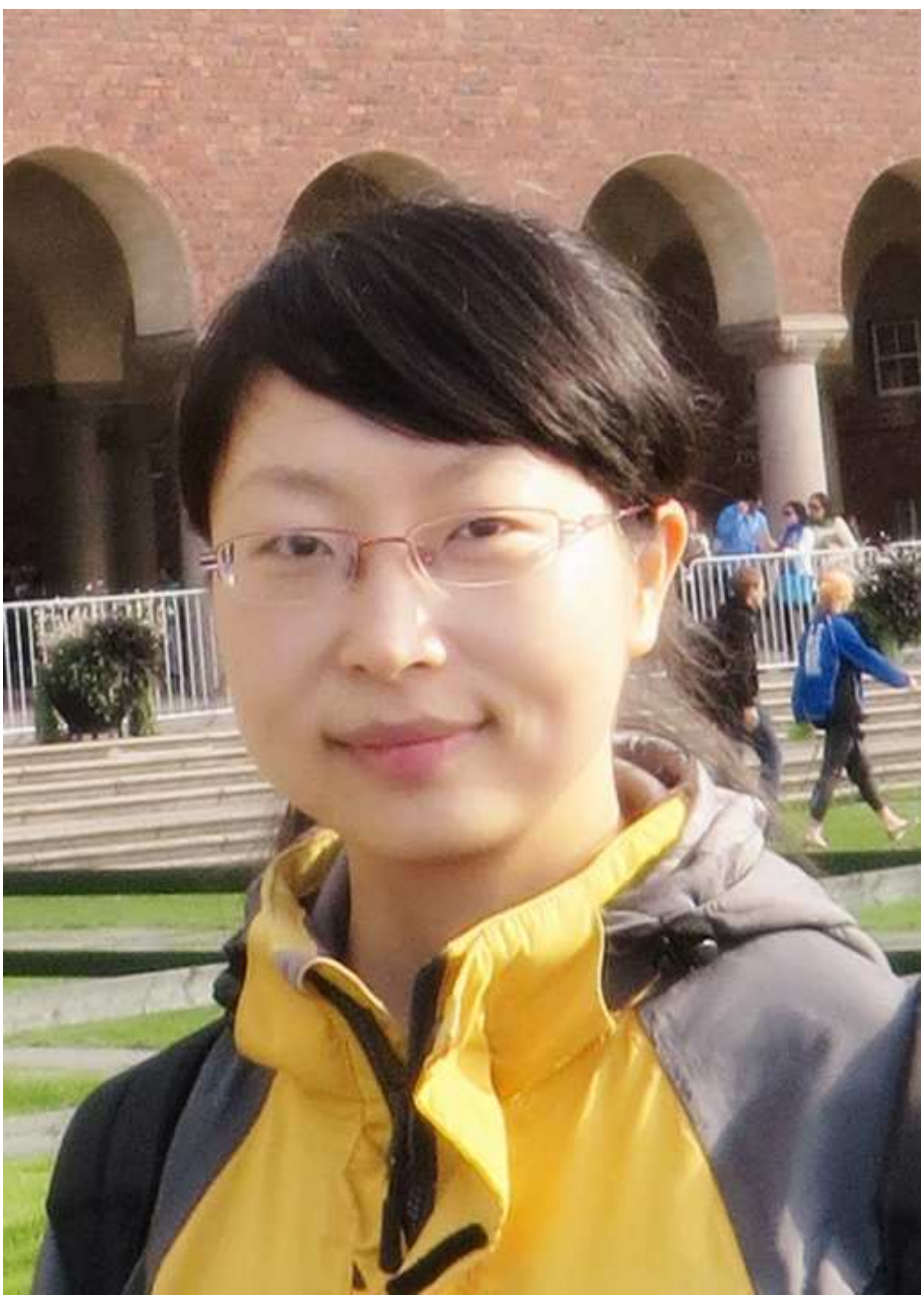


*Source Files - Latex or Word
Click here to download Source

Click here to download Source Files - Latex or Word: elsarticle-template.tex

Files - Latex or Word: elsarticle-template.tex

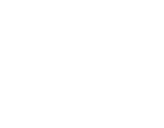
(1)

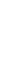

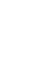
(1) (1) (1) (1) (1) (1) . . . . . . . . . . . . . . 